\title{
Decentralised final value theorem for discrete-time LTI systems with application to minimal-time distributed consensus
}

\author{
Ye Yuan ${ }^{1}$, Guy-Bart $\operatorname{Stan}^{1}$, Ling $\mathrm{Shi}^{2}$ and Jorge Gonçalves ${ }^{1}$.
}

\begin{abstract}
In this study, we consider an unknown discretetime, linear time-invariant, autonomous system and characterise, the minimal number of discrete-time steps necessary to compute the asymptotic final value of a state. The results presented in this paper have a direct link with the celebrated final value theorem. We apply these results to the design of an algorithm for minimal-time distributed consensus and illustrate the results on an example.
\end{abstract}

\section{INTRODUCTION}

Linear systems theory has played a key important role in many technology advancements in various areas like aerospace, communications, networks and computer engineering. A particular application of linear systems theory lies in the design of efficient distributed consensus algorithms and this problem has received increasingly more attention in recent years ([14], [15], [16], [17], [18] give a comprehensive introduction to this topic summarising recent developments in the multi-agent consensus problem). In these papers, the proposed algorithms ensure that each agent's state reaches consensus asymptotically. From a practical point of view however, requiring infinite (or arbitrarily long) time to obtain the final consensus value of the system is unsatisfactory. In [11], an algorithm for computing the final consensus value within a finite number of discrete-time steps is proposed based on the accumulation of a finite number of state values when the system is successively started at different initial conditions.

This paper extends the results in [11] by characterising the minimal time which is needed to compute the asymptotic final value of a state in the system using only the minimal number of successive values of this state. The paper is organised as follows: after defining our notations in Section I$\mathrm{A}$, the considered system model and problem formulation is introduced in Section I-B. Section II proposes a method, which allows to compute in finite time the final value of an arbitrarily chosen state of the considered model. These results are obtained using the final value theorem and the concept of minimal polynomial of a state. Section III contains the main results of this paper. These results allow one to characterise the minimal number of successive discrete-time values of a state necessary to reconstruct the minimal polynomial of a

\footnotetext{
${ }^{1}$ Control Group, Department of Engineering, University of Cambridge, Cambridge, U.K.. ${ }^{2}$ Department of Electronic and Computer Engineering, the Hong Kong University of Science and Technology, Hong Kong.

Emails: yy311@cam.ac.uk (Ye Yuan),gvs22@cam.ac.uk (Guy-Bart Stan), eesling@ust.hk (Ling Shi),jmg77@cam.ac.uk (Jorge Gonçalves). The authors gratefully acknowledge the support of EPSRC (project EP/E02761X/1), the support of BBSRC (project BB/D017904/1), and the support of Microsoft Research through the PhD Scholarship Programme of Mr Yuan.
}

state and further to compute the asymptotic final value of this state. Finally, an application of these theoretical results to the linear decentralised discrete-time consensus problem is proposed in Section IV. These results are contrasted with those of [2] and it is shown that the proposed method allows to compute the final value of an arbitrarily chosen state using much less information and discrete-time steps. Conclusion and future works are given in Section V.

\section{A. Notation}

For a matrix $A \in \mathbb{R}^{M \times N}, A[i, j] \in \mathbb{R}$ denotes the element in the $i^{\text {th }}$ row and $j^{\text {th }}$ column, $A[i,:] \in \mathbb{R}^{1 \times N}$ denotes its $i^{\text {th }}$ row, $A[:, j] \in \mathbb{R}^{M \times 1}$ denotes its $j^{\text {th }}$ column and $A\left[i_{1}: i_{2}, j_{1}: j_{2}\right] \in \mathbb{R}^{\left(i_{2}-i_{1}+1\right) \times\left(j_{2}-j_{1}+1\right)}$ denotes the submatrix of $A$ defined by the rows $i_{1}$ to $i_{2}$ and the columns $j_{1}$ to $j_{2}$. For a column vector $\alpha \in \mathbb{R}^{N \times 1}, \alpha[i]$ denotes its $i^{\text {th }}$ element. Similarly for a row vector $\beta \in$ $\mathbb{R}^{1 \times N}, \beta[i]$ denotes its $i^{t h}$ element. We denote by $e_{r}^{T}=$ $\left[\begin{array}{lllllll}0 & \ldots & 0 & 1_{r^{t h}} & 0 & \ldots & 0\end{array}\right] \in \mathbb{R}^{1 \times N}$.

\section{B. Problem formulation}

We consider the discrete-time LTI system

$$
\begin{aligned}
x(k+1) & =W x(k), \\
y(k) & =e_{r}^{T} x(k)=x_{r}(k)
\end{aligned}
$$

where $x(k)=\left[\begin{array}{llll}x_{1}(k) & x_{2}(k) & \ldots & x_{N}(k)\end{array}\right]^{T} \in \mathbb{R}^{N \times 1}$, $W \in \mathbb{R}^{N \times N}$, and $y(k)$ represents the observation at time $k$ of an arbitrarily chosen particular state $x_{r}(k) \in \mathbb{R}$. In the rest of this paper, we assume that both $W$ and $x(0)$ are unknown and will provide theoretical results concerning the minimal number of discrete-time steps required to compute the final value $\phi_{r}=\lim _{k \rightarrow \infty} x_{r}(k)$ of an arbitrarily chosen state $x_{r}$. Before diving into the statement and proof of the results of this paper, we first recall some fundamental results concerning Jordan block decomposition of matrices as these will be intensively used in the proofs.

\section{Polynomial of Jordan blocks}

Definition 1 (Jordan block decomposition, [1]): For any square real matrix $W \in \mathbb{R}^{N \times N}$, there exist a nonsingular matrix $S$ such that $W=S J S^{-1} \in \mathbb{R}^{N \times N}$, with $J=\left[\begin{array}{cccc}J_{1} & & & \\ & J_{2} & & \\ & & \ddots & \\ & & & J_{l}\end{array}\right], J_{i}=\operatorname{diag}\left\{J_{i 1}, J_{i 2}, \ldots, J_{i m_{i}}\right\}$, 
and $J_{i j}=\left[\begin{array}{cccc}\lambda_{i} & 1 & & \\ & \lambda_{i} & 1 & \\ & & \ddots & \ddots \\ & & & \lambda_{i}\end{array}\right] \in \mathbb{C}^{n_{i j} \times n_{i j}}$, such

that $\sum_{i=1}^{l} \sum_{j=1}^{m_{i}} n_{i j}=N$ and $\lambda_{i}, i=1, \ldots, l$ are the distinct eigenvalues of $W$. The nonsingular matrix $S$ has the following form $S=\left[\begin{array}{llll}S_{1} & S_{2} & \ldots & S_{l}\end{array}\right]$, in which $S_{i}=\left[\begin{array}{llll}S_{i 1} & S_{i 2} & \ldots & S_{i m_{i}}\end{array}\right]$ and $S_{i j}=$ $\left[\begin{array}{llll}s_{i j 1} & s_{i j 2} & \ldots & s_{i j n_{i j}}\end{array}\right]$, where $s_{i j 1}$ are the right eigenvectors of $W$,

$$
W s_{i j 1}=\lambda_{i} s_{i j 1},
$$

and $s_{i j k} \neq 0$ are defined by the following linear equations for $k \geq 2$

$$
\left(W-\lambda_{i} I\right) s_{i j k}=s_{i j(k-1)}
$$

$s_{i j k}$ are called the generalised eigenvectors of $W$.

Let $T=S^{-1}$ and assume $T=\left[\begin{array}{llll}T_{1}^{T} & T_{2}^{T} & \ldots & T_{l}^{T}\end{array}\right]^{T}$, in which $T_{i}=\left[\begin{array}{llll}T_{i 1}^{T} & T_{i 2}^{T} & \ldots & T_{i m_{i}}^{T}\end{array}\right]^{T}$ and $T_{i j}=$ $\left[\begin{array}{llll}t_{i j 1}^{T} & t_{i j 2}^{T} & \ldots & t_{i j n_{i j}}^{T}\end{array}\right]^{T}$ with the same partitions as $S^{T}$.

\section{DECENTRALISED FINAL VALUE THEOREM}

In this section, we propose an algorithm for computing the final value of an arbitrarily chosen state $x_{r}$ of eq. (1) using the minimal number of discrete-time steps, provided that the coefficients of the minimal polynomial of this state are known.

We first give some definitions about matrices and polynomials: if $p(t)=t^{k}+\beta_{k-1} t^{k-1}+\cdots+\beta_{1} t+\beta_{0}$ is a given polynomial, then one can define $p(A)=A^{k}+\beta_{k-1} A^{k-1}+$ $\cdots+\beta_{1} A+\beta_{0} I_{N}$ for any $A \in \mathbb{R}^{N \times N}$. We call the polynomial monic if the coefficient of the largest order term is 1 . We then give the definitions of the minimal polynomial of a matrix and of the minimal polynomial of a state.

Definition 2 (Minimal polynomial of a matrix): The minimal polynomial associated with a matrix $W \in \mathbb{R}^{N \times N}$ is denoted by $q(t)$ and is defined as the unique, minimal degree monic polynomial which satisfies $q(W)=0$.

Definition 3 (Minimal polynomial of a state): For a discrete time system satisfying eq. (1), the minimal polynomial associated with state $x_{r} \in \mathbb{R}$ is denoted by $q_{r}(t)$ and is defined as the unique, minimal degree monic polynomial which satisfies $e_{r}^{T} q_{r}(W)=0$.

Remark 1: The polynomial of a state is not necessarily the same as that of a matrix, moreover, $q_{r}(t)$ divides $q(t)$ (see [2] for a proof of these statements).

In the following parts of this section, we are going to characterise the explicit form of the minimal polynomial associated with an arbitrarily chosen state $x_{r}$. From eq. (1), we have

$$
x_{r}(k)=e_{r}^{T} W^{k} x(0)=e_{r}^{T} S J^{k} T x(0),
$$

where $T=S^{-1}$. Based on the Jordan block decomposition (see Section I-C), we have, for all $k$ :

$$
\begin{aligned}
J_{i j}^{k} & \left.=\left[\begin{array}{cccc}
\lambda_{i} & & & \\
& \lambda_{i} & & \\
& & \ddots & \\
& & & \lambda_{i}
\end{array}\right]+\left[\begin{array}{cccc}
0 & 1 & & \\
& 0 & 1 & \\
& & \ddots & \ddots \\
& & & 0
\end{array}\right]\right)^{k} \\
& =\left[\begin{array}{ccccc}
\lambda_{i}^{k} & \left(\begin{array}{c}
k \\
1
\end{array}\right) \lambda_{i}^{k-1} & \ldots & & \left(\begin{array}{c}
k \\
n_{i j}-1
\end{array}\right) \lambda_{i}^{k-n_{i j}} \\
& \lambda_{i}^{k} & \left(\begin{array}{c}
k \\
1
\end{array}\right) \lambda_{i}^{k-1} & \ldots & \left(\begin{array}{c}
k \\
n_{i j}-2
\end{array}\right) \lambda_{i}^{k+1-n_{i j}} \\
& & \ddots & \ddots & \vdots \\
& & & \lambda_{i}^{k} & \left(\begin{array}{c}
k \\
1
\end{array}\right) \lambda_{i}^{k-1} \\
& & & & \lambda_{i}^{k}
\end{array}\right],
\end{aligned}
$$

where

$$
\left(\begin{array}{c}
k \\
i
\end{array}\right)=\left\{\begin{array}{rr}
\frac{k !}{i !(k-i) !} & \text { for } k \geq i \\
0 & \text { otherwise }
\end{array}\right.
$$

Now, let $e_{r}^{T} S=e_{r}^{T}\left[\begin{array}{lllllll}s_{11} & s_{12} & \ldots & s_{1 m_{1}} & s_{21} & \ldots & s_{l m_{l}}\end{array}\right]$ $\triangleq \gamma^{r}=\left[\begin{array}{lllllll}\gamma_{11}^{r} & \gamma_{12}^{r} & \ldots & \gamma_{1 m_{1}}^{r} & \gamma_{21}^{r} & \ldots & \gamma_{l m_{l}}^{r}\end{array}\right] \in$ $\mathbb{R}^{1 \times N}, \quad \gamma_{i j}^{r} \in \mathbb{R}^{1 \times n_{i j}}$ and $\operatorname{Tx}(0) \triangleq \beta=$ $\left[\begin{array}{lllllll}\beta_{11}^{T} & \beta_{12}^{T} & \ldots & \beta_{1 m_{1}}^{T} & \beta_{21}^{T} & \ldots & \beta_{l m_{l}}^{T}\end{array}\right]^{T} \in \mathbb{R}^{N \times 1}$, with $\beta_{i j} \in \mathbb{R}^{n_{i j} \times 1}$. This implies that $x_{r}(k)$ in (2) could be rewritten as

$$
x_{r}(k)=\sum_{i=1}^{l}\left(\sum_{j=1}^{m_{i}} \gamma_{i j}^{r} J_{i j}^{k} \beta_{i j}\right) .
$$

Using eq. (3), each term in eq. (4) can be shown to have the following expression:

$$
\begin{aligned}
\gamma_{i j}^{r} J_{i j}^{k} \beta_{i j} & =\sum_{t=0}^{n_{i j}-1}\left(\sum_{s=1}^{n_{i j}-t} \gamma_{i j}^{r}[s] \beta_{i j}[s+t]\left(\begin{array}{c}
k \\
t
\end{array}\right) \lambda_{i}^{k-t}\right) \\
& =\sum_{h=1}^{n_{i j}} \gamma_{i j}^{r}[h] \beta_{i j}[h] \lambda_{i}^{k}+\sum_{h=1}^{n_{i j}-1} \gamma_{i j}^{r}[h] \beta_{i j}[h+1]\left(\begin{array}{c}
k \\
1
\end{array}\right) \lambda_{i}^{k-1} \\
& +\ldots+\gamma_{i j}^{r}[1] \beta_{i j}\left[n_{i j}\right]\left(\begin{array}{c}
k \\
n_{i j}-1
\end{array}\right) \lambda_{i}^{k-n_{i j}} \\
& \triangleq g_{i j}\left(\lambda_{i}, k\right)+g_{i j}^{(1)}\left(\lambda_{i}, k\right)+\ldots+g_{i j}^{\left(n_{i j-1}^{r}\right)}\left(\lambda_{i}, k\right)
\end{aligned}
$$

in which $g_{i j}(\cdot, \cdot): \mathbb{C} \times \mathbb{Z} \rightarrow \mathbb{C}$, and $g^{\left(n_{i j-1}^{r}\right)}(\cdot, \cdot)$ represents the $\left(n_{i j}-1\right)^{t h}$ derivative of $g_{i j}(\cdot, \cdot)$ with respect to $\lambda_{i}$, and $n_{i j}^{r} \leq n_{i j}$. Consider $\sum_{r=1}^{n_{i j}-1} \sum_{j=1}^{m_{i}} g_{i j}^{(r)}\left(\lambda_{i}, k\right)=G_{i}\left(\lambda_{i}, k\right)$ and notice that $\left(\begin{array}{c}k \\ n_{i j}^{r}-1\end{array}\right)=\frac{k(k-1) \ldots\left(k+2-n_{i j}^{r}\right)}{\left(n_{i j}^{r}-1\right)\left(n_{i j}^{r}-2\right) \ldots 1} \propto k^{n_{i j}^{r}-1}$. This implies that $G_{i}\left(\lambda_{i}, k\right) \propto k^{n_{i}^{r}-1}$, in which $n_{i}^{r}=\max _{j}\left\{n_{i j}^{r}\right\}$. Based on eq. (5), eq. (4) becomes

$$
x_{r}(k)=\sum_{i=1}^{l} G_{i}\left(\lambda_{i}, k\right) \triangleq \sum_{i=1}^{l_{r}} v_{i}^{T}(k) \hat{\boldsymbol{G}}_{i}
$$

in which $\hat{\boldsymbol{G}}_{i} \in \mathbb{R}^{n_{i}^{r}}$ is a constant vector containing the parameters of $G_{i}\left(\lambda_{i}, k\right), l_{r} \leq l$ (because some $\hat{\boldsymbol{G}}_{i}(k)$ could 
be 0$)$ and $v_{i}^{T}(k) \triangleq\left[\begin{array}{llll}\lambda_{i}^{k} & k \lambda_{i}^{k-1} & \ldots & k^{n_{i}^{r}-1} \lambda_{i}^{k-n_{i}^{r}+1}\end{array}\right]$. Let $V(0, k) \triangleq\left[\begin{array}{cccc}v_{1}^{T}(0) & v_{2}^{T}(0) & \ldots & v_{l_{l}}^{T}(0) \\ v_{1}^{T}(1) & v_{2}^{T}(1) & \ldots & v_{l_{r}}^{T}(1) \\ \vdots & \vdots & \ddots & \vdots \\ v_{1}^{T}(k) & v_{2}^{T}(k) & \ldots & v_{l_{r}}^{T}(k)\end{array}\right] \in \mathbb{C}^{(k+1) \times\left(D_{r}+1\right)}$,

in which $D_{r}+1=\sum_{i=1}^{l_{r}} n_{i}^{r} . V(0, k)$ is a confluent Vandermonde matrix, which satisfies the following property (see [3]): $V\left(0, D_{r}\right)=\left[\begin{array}{llll}E_{r} & J_{r}^{T} E_{r} & \ldots & \left(J_{r}^{D_{r}}\right)^{T} E_{r}\end{array}\right]^{T}$, in which $J_{r}$ is the Jordan block corresponding to the eigenvalues $\lambda_{i}$ in eq. (6), $E_{r}^{T}=\left[e^{\left[m_{1}\right] T}, \ldots e^{\left[m_{l_{r}}\right] T}\right]$ is a vector partitioned according to $J_{r}, e^{\left[m_{i}\right] T}=\left[\begin{array}{llll}1, & 0, \ldots, 0\end{array} \in\right.$ $\mathbb{R}^{1 \times m_{i}}$, and $V(0, k)[k+1,:]=E_{r}^{T} J_{r}^{k}$. Let $\boldsymbol{h}_{r}^{T}=$ $\left[\begin{array}{llll}\hat{\boldsymbol{G}}_{1}^{T} & \ldots & \hat{\boldsymbol{G}}_{l_{r}-1}^{T} & \hat{\boldsymbol{G}}_{l_{r}}^{T}\end{array}\right] \in \mathbb{R}^{1 \times\left(D_{r}+1\right)} \cdot \boldsymbol{h}_{r}^{T}$ is thus also a constant vector and we have:

$$
x_{r}(k)=V(0, k)[k+1,:] \boldsymbol{h}_{r}=E_{r}^{T} J_{r}^{k} \boldsymbol{h}_{r}, \forall k .
$$

Theorem 1: Consider the discrete-time LTI system given in eq. (1). The minimal polynomial associated with state $x_{r}$, and defined in Definition 3, is the same as the characteristic polynomial of the matrix $J_{r}$ appearing in eq. (8). The final value of $x_{r}$, i.e., $\phi_{r}$ can be computed based on the coefficients of the minimal polynomial of $x_{r}$, and on the successive values of $x_{r}$ as described in eq. (11).

Proof: The Jordan matrix $J_{r}$ appearing in eq. (8) has the following property: each of its Jordan block has distinct eigenvalues. Due to this latter property, the minimal polynomial of $x_{r}$ is the same as the characteristic polynomial of $J_{r}$ [1]. Therefore, this minimal polynomial possesses the following explicit form: $q_{r}\left(J_{r}\right)=\operatorname{det}\left(J_{r}-t I\right)=\prod_{i=1}^{l_{r}}(t-$ $\left.\lambda_{i}\right)^{n_{i}^{r}}=J_{r}^{D_{r}+1}+\alpha_{D_{r}} J_{r}^{D_{r}}+\ldots+\alpha_{1} J_{r}+\alpha_{0}=0$, and has degree $D_{r}+1$.

From eq. (8), we then obtain

$$
\begin{aligned}
x_{r}\left(D_{r}+1\right) & =E_{r} J_{r}^{D_{r}+1} \boldsymbol{h}_{r} \\
& =-E_{r}\left(\alpha_{D_{r}} J_{r}^{D_{r}}+\ldots+\alpha_{1} J_{r}+\alpha_{0} I\right) \boldsymbol{h}_{r} \\
& =-\alpha_{D_{r}} x_{r}\left(D_{r}\right)-\ldots-\alpha_{1} x_{r}(1)-\alpha_{0} x_{r}(0) .
\end{aligned}
$$

Since this latter equation holds for all $\boldsymbol{h}_{r}$ (which is dependent of the initial condition $x(0)$ ), the following linear difference equations must be satisfied $\forall k \geq 0$ :

$x_{r}\left(k+D_{r}+1\right)+\alpha_{D_{r}} x_{r}\left(k+D_{r}\right)+\ldots+\alpha_{1} x_{r}(k+1)+\alpha_{0} x_{r}(k)=0$

Using the Jury stability criterion for polynomials [8], we can check whether the polynomial defined in eq. (10) possesses at least an unstable root, i.e., a root, different form 1 and such that its magnitude is larger or equal to 1 . If $q_{r}(t)=$ 0 possesses at least one unstable root, then we know that $\phi_{r}=\lim _{k \rightarrow \infty} x_{r}(k)=\infty$. On the contrary, if all the roots of $q_{r}(t)=0$ are stable, then $\phi_{r}=0$. Furthermore, as shown in [2], under the assumption that the minimal polynomial in (10) does not possess any unstable root, except for one single root located at 1 , we can take the Z-transform of (10) and apply the final value theorem to compute the final value of $x_{r}$ based on the coefficient of the minimal polynomial $q_{r}(\cdot)$ :

$$
\begin{aligned}
\lim _{k \rightarrow \infty} x_{r}(k) & =\lim _{z \rightarrow 1}(z-1) X_{r}(z) \\
& =\frac{\left[\begin{array}{llll}
x_{r}\left(D_{r}\right) & x_{r}\left(D_{r}-1\right) & \ldots & x_{r}(0)
\end{array}\right] S}{\left[\begin{array}{llll}
1 & 1 & \ldots & 1
\end{array}\right] S}
\end{aligned}
$$

in which $S=\left[\begin{array}{c}1 \\ 1+\alpha_{D_{r}} \\ 1+\alpha_{D_{r}-1}+\alpha_{D_{r}} \\ \vdots \\ 1+\sum_{j=1}^{D_{r}} \alpha_{j}\end{array}\right]$.

In the rest of the paper, we make the following assumption:

Assumption 1: $q_{r}(t)=0$ does not possess any root such that its magnitude is larger or equal to 1, except, potentially, for one single root located at 1.

Remark 2: Once the coefficients of the minimal polynomial of $x_{r}$ are known the result in eq. (11) shows that, under Assumption 1, the final value of $x_{r}$ can be obtained as a linear combination of the first $D_{r}+1$ successive values of $x_{r}$ obtained according to eq. (1) starting from the initial condition $x_{r}(0)$, where $D_{r}+1$ is the degree of the minimal polynomial of $x_{r}$.

Remark 3: A consequence of Theorem 1 is that the roots of $q_{r}(t)=0$ are the eigenvalues of the Jordan matrix $J_{r}$ appearing in (8).

In the following section, we propose an algorithm for computing the coefficients of the minimal polynomial of $x_{r}$ using the least number of successive values of $x_{r}$.

\section{Computation of THE COEFFicients of THE MINIMAL POLYNOMIAL OF $x_{r}$}

In the previous section, we showed that the final value of an arbitrarily chosen state $x_{r}$ of a discrete-time, LTI system can be computed in minimal time based on the knowledge of the coefficients of the minimal polynomial of $x_{r}$ (see eq. (11)). In this section, we first provide centralised results characterising the minimal number of successive discretetime values of $x_{r}$ needed to compute the coefficients of the minimal polynomial of $x_{r}$ in eq. (10). We then propose a decentralised algorithm for obtaining these coefficients using the minimal number of successive discrete-time values of $x_{r}$.

\section{A. Centralised computation of the coefficients of the minimal polynomial of $x_{r}$}

0 . In this subsection, we assume knowledge by the arbitrarily chosen state $x_{r}$ of the order $D_{r}+1$ of its associated minimal polynomial $q_{r}(\cdot)$ and of the multiplicity $\delta_{r}$ of the root 0 of the minimal polynomial of $x_{r}$. For this reason, the results presented in this subsection are considered "centralised". In the next subsection, we propose a decentralised algorithm which does not require the knowledge of $D_{r}+1$ and $\delta_{r}$.

Theorem 2: Consider the model in eq. (1). Assuming that the arbitrarily chosen state $x_{r}$ knows the order and the multiplicity of the root 0 of its associated minimal polynomial and that Assumption 1 is satisfied, the minimal number of successive discrete-time values (starting from the discrete-time instant $i$ ) of an arbitrarily chosen state $x_{r}$ 
necessary to compute the minimal polynomial coefficients $\alpha_{0}, \alpha_{1}, \ldots, \alpha_{D_{r}}$ appearing in eq. (10) is $2\left(D_{r}+1\right)-\delta_{r}-$ $\min \left\{i, \delta_{r}\right\}$.

Proof: By definition of 0 as a root of the minimal polynomial, we have $a_{0}=\cdots=a_{\delta_{r}-1}=0$ and the first $\delta_{r}$ values of $x_{r}$, i.e., $x_{r}(0), \ldots, x_{r}\left(\delta_{r}-1\right)$ are useless for the computation of the coefficients of the minimal polynomial $q_{r}(\cdot)$. Eq. (10) thus writes $\alpha_{\delta_{r}} x_{r}\left(k+\delta_{r}\right)+\alpha_{\delta_{r}+1} x_{r}(k+$ $\left.\delta_{r}+1\right)+\ldots+\alpha_{D_{r}} x_{r}\left(k+D_{r}\right)+x_{r}\left(k+D_{r}+1\right)=0$. Because there are $D_{r}+1-\delta_{r}$ unknowns in this equation, i.e., $\alpha_{\delta_{r}}, \ldots, \alpha_{D_{r}}$, we need $D_{r}+1-\delta_{r}$ independent equations. These equations are given by:

$\forall k=i, i+1, \ldots i+D_{r}-\delta_{r}$ :

$x_{r}\left(k+D_{r}+1\right)+\alpha_{D_{r}} x_{r}\left(k+D_{r}\right)+\ldots+\alpha_{\delta_{r}} x_{r}\left(k+\delta_{r}\right)=0$.

Since these equations can easily be shown to be linearly independent (see [9] for more details), the minimal number of successive discrete-time values of $x_{r}$ (starting from $x_{r}(i)$ ) which are needed to compute the nonzero coefficients of the minimal polynomial of $x_{r}$ is $2\left(D_{r}+1\right)-\delta_{r}-\min \left\{i, \delta_{r}\right\}$.

\section{B. Decentralised computation of the coefficients of the min- imal polynomial of $x_{r}$}

In this section, we assume that the computation of the final value of $x_{r}$ must be realised in a purely decentralised way, i.e., without access to any kind of centralised information such as the order $D_{r}+1$ of the minimal polynomial of $x_{r}$ The problem is defined as follows:

Definition 4 (Decentralised problem): Consider the linear dynamics in eq. (1). The goal for an arbitrarily chosen state $x_{r}$ is to compute its final asymptotic value $\phi_{r}=$ $\lim _{k \rightarrow \infty} x_{r}(k)$ using only its own previously observed values. In particular, we assume $x_{r}$ does not have access to any other external information such as the degree of its minimal polynomial $D_{r}+1$, or the total number of states $N$ in the dynamics (1).

To determine the coefficients of the minimal polynomial of $x_{r}$, we will use a particular Hankel matrix $\boldsymbol{X}_{r}\left(k_{1}, k_{2}\right) \in$ $\mathbb{R}^{\left(k_{1}+1\right) \times\left(k_{2}+1\right)}\left(k_{1}, k_{2} \in \mathbb{N}\right)$ containing the values of $x_{r}$ at successive discrete time instants.

$$
\boldsymbol{X}_{r}\left(k_{1}, k_{2}\right) \triangleq\left[\begin{array}{cccc}
x_{r}(0) & x_{r}(1) & \ldots & x_{r}\left(k_{2}\right) \\
x_{r}(1) & \left.x_{r}(2)\right) & \ldots & x_{r}\left(k_{2}+1\right) \\
x_{r}(2) & \ddots & & x_{r}\left(k_{2}+2\right) \\
\vdots & \vdots & \ddots & \vdots \\
x_{r}\left(k_{1}\right) & x_{r}\left(k_{1}+1\right) & \ldots & x_{r}\left(k_{1}+k_{2}\right)
\end{array}\right] .
$$

Theorem 3: Consider the model in eq. (1). If Assumption 1 is satisfied, the minimal number of successive discretetime values (starting from the discrete-time instant 0) of an arbitrarily chosen state $x_{r}$ necessary to compute the minimal polynomial coefficients $\alpha_{0}, \alpha_{1}, \ldots, \alpha_{D_{r}}$ appearing in eq. (10) is $2 \rho_{r}+1=2 D_{r}+3$, where $\rho_{r}$ is the maximal rank of $\boldsymbol{X}_{r}$ for increasing values of its dimensions, i.e., $\rho_{r} \triangleq \operatorname{rank} \boldsymbol{X}_{r}\left(D_{r}, D_{r}\right)=\operatorname{rank} \boldsymbol{X}_{r}\left(D_{r}+1, D_{r}+1\right)=\cdots=$ $\operatorname{rank} \boldsymbol{X}_{r}(\infty, \infty)$. The coefficients of the minimal polynomial associated with $x_{r}$ are then obtained by computing the kernel of the Hankel matrix $\boldsymbol{X}_{r}\left(D_{r}+1, D_{r}+1\right)$, i.e., $\boldsymbol{\alpha}_{r}=$ $\left[\begin{array}{lllll}\alpha_{0} & \alpha_{1} & \ldots & \alpha_{D_{r}} & 1\end{array}\right]^{T} \in \operatorname{ker}\left\{\boldsymbol{X}_{r}\left(D_{r}+1, D_{r}+1\right)\right\}$.

Proof: From eq. (8), it is easy to show that the Hankel matrix in (12) has the following decomposition

$$
\boldsymbol{X}_{r}\left(k_{1}, k_{2}\right)=\left[\begin{array}{c}
E_{r}^{T} \\
E_{r}^{T} J_{r} \\
\vdots \\
E_{r}^{T} J_{r}^{k_{1}}
\end{array}\right]\left[\begin{array}{llll}
\boldsymbol{h}_{r} & J_{r} \boldsymbol{h}_{r} & \ldots & J_{r}^{k_{2}} \boldsymbol{h}_{r}
\end{array}\right] .
$$

where $E_{r}^{T}, J_{r}$ and $\boldsymbol{h}_{r}$ are defined in eq. (8). Based on the decomposition in eq. (13), we can characterise the minimal dimensions $k_{1, \min }$ and $k_{2, \min }$ necessary to the computation of the coefficients of the minimal polynomial of $x_{r}$.

Without loss of generality, we start by characterising the minimal value of $k_{2}$. In order to do so, we fix $k_{1}$ and assume that $d_{r}+1$ is the smallest integer for which the Hankel matrix $\boldsymbol{X}_{r}\left(k_{1}, d_{r}+1\right)$ loses its column rank. Consequently, there exists a vector $\boldsymbol{a}_{r}=\left[\begin{array}{lllll}a_{0} & a_{1} & \ldots & a_{d_{r}} & 1\end{array}\right]^{T}$ such that $\boldsymbol{X}_{r}\left(k_{1}, d_{r}+1\right) \boldsymbol{a}_{r}=\mathbf{0}$. This, in turn, implies that $\left[\begin{array}{llll}\boldsymbol{h}_{r} & J_{r} \boldsymbol{h}_{r} & \ldots & J_{r}^{d_{r}+1} \boldsymbol{h}_{r}\end{array}\right] \boldsymbol{a}_{r}=0$, which is equivalent to

$$
a_{0} \boldsymbol{h}_{r}+a_{1} J_{r} \boldsymbol{h}_{r}+\ldots+a_{d_{r}} J_{r}^{d_{r}} \boldsymbol{h}_{r}+J_{r}^{d_{r}+1} \boldsymbol{h}_{r}=0 .
$$

Since eq. (14) must hold $\forall \boldsymbol{h}_{r}$, the following equation must be satisfied $a_{0}+a_{1} J_{r}+\ldots+a_{d_{i}} J_{r}^{d_{r}}+J^{d_{r}+1} \equiv 0$. By definition of the minimal polynomial of state $x_{r}$, the minimal $d_{r}$ must thus be $D_{r}$ and $\boldsymbol{a}_{r}=\boldsymbol{\alpha}_{r}$.

We now characterise the minimal value of $k_{1}$. For that, we assume that $k_{2}$ is fixed to its minimal value computed above, i.e., $k_{2}=k_{2, \min }=D_{r}+1$, and denote by $\tilde{d}_{r}+1$ the smallest integer which satisfies the following property: if $\boldsymbol{X}_{r}\left(\tilde{d}_{r}+\right.$ $\left.1, D_{r}+1\right) \boldsymbol{\alpha}_{r}=\mathbf{0}$, then $\forall \tilde{d} \geq \tilde{d}_{r}, \boldsymbol{X}_{r}\left(\tilde{d}+1, D_{r}+1\right) \boldsymbol{\alpha}_{r}=\mathbf{0}$. Using the definitions of $\boldsymbol{X}_{r}$ in (12) and the linear difference eq. (10), it is easy to see that this latter property holds $\forall \tilde{d}_{r} \geq$ $D_{r}-1$. However, in order to compute $D_{r}$, we need to check for which value of $D_{r} \operatorname{rank} \boldsymbol{X}_{r}\left(D_{r}, D_{r}\right)=\operatorname{rank} \boldsymbol{X}_{r}\left(D_{r}+\right.$ $\left.1, D_{r}+1\right)$. The construction of $\boldsymbol{X}_{r}\left(D_{r}+1, D_{r}+1\right)$ requires knowledge of the values of $x_{r}(0), \ldots, x_{r}\left(2 D_{r}+2\right)$. Once the value of $D_{r}$ is obtained, the coefficients of the minimal polynomial can be computed by looking at the kernel of the matrix $\boldsymbol{X}_{r}\left(D_{r}+1, D_{r}+1\right)$, i.e., $\boldsymbol{X}_{r}\left(D_{r}+1, D_{r}+1\right) \boldsymbol{\alpha}_{r}=0$.

The analysis above shows that $k_{1, \text { min }}+k_{2, \text { min }}+1=$ $\left(D_{r}+1\right)+\left(D_{r}+1\right)+1=2 D_{r}+3=2 \rho_{r}+1$ with $\rho_{r}=$ $\operatorname{rank} \boldsymbol{X}_{r}\left(D_{r}, D_{r}\right)$ since $\boldsymbol{X}_{r}\left(D_{r}, D_{r}\right)$ is full rank. Therefore, the minimal number of consecutive state values of $x_{r}$ needed to reconstruct the coefficient of the minimal polynomial of $x_{r}$ is $2 D_{r}+3$.

Theorem 3 gives a simple decentralised algorithm for computing the coefficients of the minimal polynomial of $x_{r}$ based on square Hankel matrices of the form given in eq. (12).

Corollary 1: Consider the model in eq. (1). For any state $x_{r}$ such that Assumption 1 is satisfied, the number of successive values that must be considered in order to be able to compute the coefficients of the minimal polynomial of $x_{r}$ is upper bounded by $2 N+1$. This latter upper bound is achieved when the minimal polynomials of all states are the same as the characteristic polynomial of $W$, and $W$ has no 0 eigenvalues. 
Proof: It can easily be shown from the definition and the fact $D_{r}+1 \leq N$.

The above proposed coefficient-reconstruction algorithm does not depend on the starting discrete-time instant of $x_{r}$ if the minimal polynomial of state $x_{r}$ does not have any root at 0 . Therefore, we could start at any arbitrary discrete-time $k$ and follow the same procedure. However, if the minimal polynomial of $x_{r}$ has a root of multiplicity $\delta_{r}$ at 0 , then this can be exploited in order to reduce the necessary number of successive values of $x_{r}$ as summarised in Corollary 2 .

Corollary 2: Consider the model in eq. (1). Assuming that Assumption 1 is satisfied, the minimal number of successive discrete-time values starting from discrete-time instant $i$ of an arbitrarily chosen state $x_{r}$ necessary to compute the minimal polynomial coefficients appearing in eq. (10) is $2\left(D_{r}+1\right)-$ $\delta_{r}-\min \left\{i, \delta_{r}\right\}+1$.

Proof: The proof is based a similar approach as the one used in the proof of Theorem 3. Notice that

$$
\begin{aligned}
& \boldsymbol{X}_{r}\left(D_{r}+1, D_{r}+1\right) \boldsymbol{\alpha}_{r} \\
& =\boldsymbol{X}_{r}\left(D_{r}+1, D_{r}+1\right)\left[:, \delta_{r}+1: D_{r}+2\right] \hat{\boldsymbol{\alpha}}_{r}
\end{aligned}
$$

where $\boldsymbol{\alpha}_{r}=\left[\begin{array}{lllllll}\underbrace{\alpha_{0}}_{=0} & \cdots & \underbrace{\alpha_{\delta_{r}-1}}_{=0} & \alpha_{\delta_{r}} & \ldots & \alpha_{D_{r}} & 1\end{array}\right]^{T}$ and $\hat{\boldsymbol{\alpha}}_{r}=\left[\begin{array}{llll}\alpha_{\delta_{r}} & \ldots & \alpha_{D_{r}} & 1\end{array}\right]^{T}$. We then note that in a decentralised problem setting (see Definition 4), $k_{1, \min }$ should be chosen the same as $k_{2, \text { min }}$. Therefore it is easy to see that the minimal number of successive values of $x_{r}$ corresponding to $\boldsymbol{X}_{r}\left(D_{r}+1, D_{r}+1\right)\left[\delta_{r}+1: D_{r}+2, \delta_{r}+1\right.$ : $\left.D_{r}+2\right]$ is $2\left(D_{r}+1-\delta_{r}\right)+1$. Similarly to the proof of Theorem 2, we can easily show that the successive values corresponding to $i<\delta_{r}$, i.e., $x_{r}(0), \ldots, x_{r}\left(\delta_{r}-1\right)$ are useless for the computation of the coefficients. Therefore the minimal number of successive value of $x_{r}$ is $2\left(D_{r}+1\right)-$ $\delta_{r}-\min \left\{i, \delta_{r}\right\}+1$.

Example 1: We consider an autonomous LTI discrete-time system for which the minimal polynomial of the arbitrarily chosen state $x_{r}$ is of degree 3 and has the roots $\{0,1,1 / 2\}$. The corresponding Hankel matrix composed of the successive values $x_{r}(0), \ldots, x_{r}(6)$ is given by:

$$
\boldsymbol{X}_{r}(3,3)=\left[\begin{array}{cccc}
1 / 6 & 2 / 3 & 7 / 12 & 13 / 24 \\
2 / 3 & 7 / 12 & 13 / 24 & 25 / 48 \\
7 / 12 & 13 / 24 & 25 / 48 & 49 / 96 \\
13 / 24 & 25 / 48 & 49 / 96 & 97 / 192
\end{array}\right]
$$

Noting that $\operatorname{rank} \boldsymbol{X}_{r}(3,3)=\operatorname{rank} \boldsymbol{X}_{r}(2,2)=3$, we need $2\left(D_{r}+1\right)+1=2 \times 3+1=7$ steps to compute the coefficients of the minimal polynomial of $x_{r}$. However, if we build the Hankel matrix using successive values of $x_{r}$ which start from the discrete time 2 , then $\operatorname{rank} \boldsymbol{X}_{r}(3,3)[2$ : $4,2: 4]=\operatorname{rank} \boldsymbol{X}_{r}(3,3)[2: 3,2: 3]=2$, that is to say, we only need $2 \times(3-1)+1=5$ steps to reconstruct the coefficients.

Remark 4: Even if $W$ contains some unstable eigenvalues, some states may still converge to a finite asymptotic value if their corresponding minimal polynomials do not have any unstable roots. For example, consider $W=\left[\begin{array}{ll}2 & 0.1 \\ 0 & 0.5\end{array}\right]$ in (1). The corresponding linear dynamics is unstable. However,

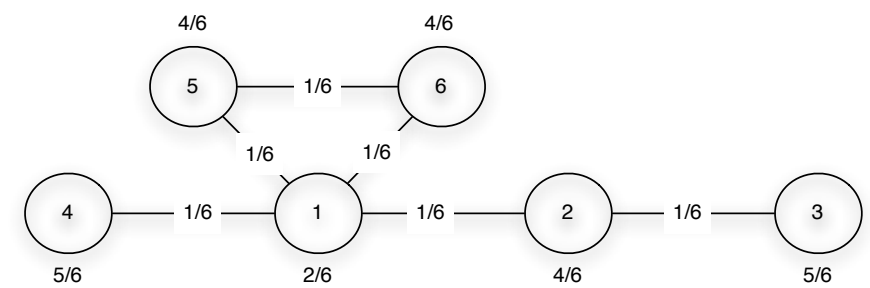

Fig. 1. Graph with constant edge weights of $1 / N$.

the state $x_{2}(k)=0.5^{k} x_{2}(0)$ converges to a finite final value as $k \rightarrow \infty$ because its associated minimal polynomial does not have any unstable roots.

Finally, we propose a complete algorithm to compute the final value $\phi_{r}=\lim _{k \rightarrow \infty} x_{r}(k)$ using the minimal number of successive values of the observed state $x_{r}$. For simplicity, we are using the successive values of $x_{r}$ starting from $x_{r}(0)$ and assume that $q_{r}(t)=0$ does not have any 0 root.

Step 1: Increase the dimension $k$ of the square Hankel matrix $\boldsymbol{X}_{r}(k, k)$ until it loses rank and store the first defective Hankel matrix.

Step 2: The $\left[\begin{array}{lllll}\alpha_{0} & \alpha_{1} & \ldots & \alpha_{D_{r}} & 1\end{array}\right]^{T}$ of the first defective Hankel matrix gives the coefficients of eq. (10).

Step 3: Compute the final value $\phi_{r}$ using Theorem 1.

\section{ApPLICATIONS}

In this section, we apply the results presented in the previous sections to the distributed asymptotic consensus problem defined in Definition 5. In particular, we show that each node can immediately compute the consensus value after observing and storing the evolution of its own value over a finite and minimal number of discrete-time steps.

Definition 5 (Distributed asymptotic consensus): The system (1) is said to asymptotically achieve distributed consensus if

$$
\lim _{k \rightarrow \infty} x(k)=\mathbf{1} c^{T} x(0)
$$

where $\mathbf{1}$ is the column vector with all components equal to 1 , and $c^{T}$ is some constant row vector. In other words, the values of all nodes converge to the same linear combination of the initial node values $c^{T} x(0)$.

As shown by [7], the distributed consensus problem formulated in Definition 5 with $c$ normalised so that $c^{T} \mathbf{1}=\mathbf{1}$, can be solved under the following assumptions:

1) $W$ has a simple eigenvalue at 1 , and all other eigenvalues have a magnitude strictly less than 1 .

2) The left and right eigenvectors of $W$ corresponding to the eigenvalue 1 are $c^{T}$ and $\mathbf{1}$, respectively.

In [2], it has been further shown that if the matrix $W$ satisfies the above mentioned conditions, then each agent can compute its final consensus value in a finite number of discrete-time steps.

In order to give a illustrative example, we are going to compare our algorithm with the one proposed in [2].

Example 2: The topology we are considering is represented in Fig. 1. This topology is the same as the one used 
in [2] and will be used here to contrast our results with those of [2]. In [2], the method proposed to compute the coefficients of the minimal polynomial of $x_{r}$ requires $D_{r}+1$ successive values of $x_{r}$ per iteration with a total number of $N+1$ independent iterations, i.e., a total of $\left(D_{r}+1\right)(N+1)$ successive values of $x_{r}$. In contrast, Theorem 3 of this paper shows that the minimal number of successive values of $x_{r}$ is $2\left(D_{r}+1\right)+1$ (see Table I for a comparison of the number of successive values that must be considered for each node). Furthermore, contrary to what is assumed in [2, Section V],

\begin{tabular}{|c|c|c|}
\hline & {$[2]$} & Theorem 3 \\
\hline$N_{1}$ & $4 \times 7=28$ & $2 \times 4+1=9$ \\
\hline$N_{2}$ & $4 \times 7=28$ & $2 \times 4+1=9$ \\
\hline$N_{3}$ & $4 \times 7=28$ & $2 \times 4+1=9$ \\
\hline$N_{4}$ & $5 \times 7=30$ & $2 \times 5+1=11$ \\
\hline$N_{5}$ & $6 \times 7=36$ & $2 \times 6+1=13$ \\
\hline$N_{6}$ & $6 \times 7=36$ & $2 \times 6+1=13$ \\
\hline
\end{tabular}

COMPARISON OF THE NUMBER OF SUCCESSIVE VALUES $N_{i}$ NEEDED FOR NODE $i$ TO COMPUTE ITS FINAL VALUE.

Theorem 3 does not require that the arbitrarily chosen state $x_{r}$ knows to the total number of nodes present in the network, or any other kind of global (centralised) information about the network. The only assumption is that a node is able to store his own $2\left(D_{r}+1\right)+1$ previously observed states, where $D_{r}$ is the maximal number such that the corresponding Hankel matrix $\boldsymbol{X}_{r}\left(D_{r}, D_{r}\right)$ defined in eq. (12) is full rank.

Because the topology is undirected and connected, the final value of each node is the average of the initial state values (average consensus value, see [15]). For the randomly chosen the initial state $x(0)=$ $\left[\begin{array}{llllll}1.3389 & 2.0227 & 1.9872 & 6.0379 & 2.7219 & 1.9881\end{array}\right]^{T}$, the final consensus value is thus 2.6828. We illustrate the results presented in Theorem 3 by focusing on node 1 step by step:

Step 1:

$$
\begin{aligned}
\boldsymbol{X}_{1}(3,3)= & {\left[\begin{array}{lllll}
1.3389 & 2.5747 & 2.7797 & 2.8164 \\
2.5747 & 2.7797 & 2.8164 & 2.8211 \\
2.7797 & 2.8164 & 2.8211 & 2.8174 \\
2.8164 & 2.8211 & 2.8174 & 2.8107
\end{array}\right], } \\
\boldsymbol{X}_{1}(4,4)= & {\left[\begin{array}{lllll}
1.3389 & 2.5747 & 2.7797 & 2.8164 & 2.8211 \\
2.5747 & 2.7797 & 2.8164 & 2.8211 & 2.8174 \\
2.7797 & 2.8164 & 2.8211 & 2.8174 & 2.8107 \\
2.8164 & 2.8211 & 2.8174 & 2.8107 & 2.8029 \\
2.8211 & 2.8174 & 2.8107 & 2.8029 & 2.7946
\end{array}\right] . }
\end{aligned}
$$

Because $\operatorname{rank} \boldsymbol{X}_{1}(3,3)=\operatorname{rank} \boldsymbol{X}_{1}(4,4)=4$, we store $\boldsymbol{X}_{1}(4,4)$.

Step 2: We obtain the coefficients of the minimal polynomial of $x_{1}$ by computing the normalised kernel of $X_{1}(4,4) \quad\left[\begin{array}{lllll}0.0833 & -0.8611 & 2.4444 & -2.6667 & 1\end{array}\right]^{T}$. The minimal polynomial of $x_{1}$ is therefore $q_{1}(t)=$ $t^{4}-2.6667 t^{3}+2.4444 t^{2}-0.8611 t+0.0833$, which is the same as the one given in [2, Section V].

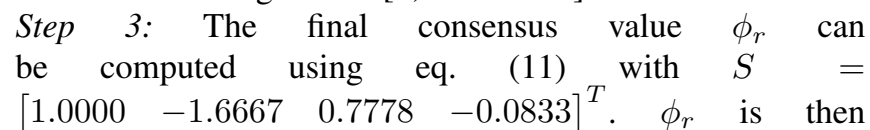

given by

$$
\begin{aligned}
& \phi_{r}=\frac{\left[\begin{array}{llll}
x_{1}(3) & x_{1}(2) & x_{1}(1) & x_{1}(0)
\end{array}\right] S}{\left[\begin{array}{llll}
1 & 1 & \ldots & 1
\end{array}\right] S} \\
& =\frac{\left[\begin{array}{llll}
2.8164 & 2.7797 & 2.5747 & 1.3389
\end{array}\right] S}{\left[\begin{array}{llll}
1 & 1 & \ldots & 1
\end{array}\right] S} \\
& =2.6828 \text {. }
\end{aligned}
$$

\section{CONCLUSION, DISCUSSION AND FUTURE WORK}

As a major contribution of this paper, we propose a decentralised algorithm enabling a node to compute its final value using only the minimal number of successive values of itself. The information at the disposal of a node is minimal as well since we do not require any global knowledge, e.g., the total number of nodes in the system, or the specific weights of the links.

A more general linear system model is considered and discussed in detail in [9] and [10]. Future work will investigate extensions of the presented results to linear time-varying discrete-time system and LTI discrete-time system perturbed with noise.

\section{REFERENCES}

[1] R. Horn and C. Johnson, Matrix analysis. Cambridge University Press 1999.

[2] S. Sundaram and C. N. Hadjicostis, "Finite-Time Distributed Consensus in Graphs with Time-Invariant Topologies," Proceedings of the American Control Conference, 2007.

[3] D. Boley, F. Luk and D. Vandevoorde, "Vandermonde Factorization of a Hankel Matrix," Scientific computing, pp. 27-39, Springer, 1997.

[4] A. Bjorck and V. Pereyra, "Solution of Vandermonde Systems of Equations," Mathematics of Computation, vol. 24, no. 112, 1970.

[5] R. H. Schappelle, "The Inverse of the Confluent Vandermonde Matrix," IEEE Transaction on Automatic Control, 1972.

[6] K. Zhou, J. Doyle and K. Glover, "Robust and Optimal Control," Prentice Hall, 1996.

[7] L. Xiao and S. Boyd, "Fast linear iterations for distributed averaging," System and control letter, vol. 53, no. 1, pp. 65-78, 2004.

[8] E. I. Jury, "Inners and stability of dynamic systems," John Wiley and Sons, 1974.

[9] Y. Yuan, G. Stan, L. Shi and J. Gonçalves, in preparation for IEEE Transaction on Automatic Control.

[10] Y. Yuan, "Final value theorem for discrete-time unknown LTI systems," M.Phil. Thesis, 2009.

[11] S. Sundaram and C. N. Hadjicostis, "Distributed Function Calculation and Consensus using Linear Iterative Strategies." IEEE Journal on Selected Areas in Communications: Issue on Control and Communications, vol. 26, no. 4, May 2008.

[12] S. Sundaram and C. N. Hadjicostis, "Distributed Calculation of Linear Functions in Noisy Networks via Linear Iterations." in Proceedings of IEEE Conference on Decision and Control, Cancun, Mexico, 2008.

[13] W. Ren and R. W. Beard, "Consensus seeking in multi-agent systems under dynamically changing interaction topologies," IEEE Transactions on Automatic Control, vol. 50, no. 5, pp. 655-661, 2005.

[14] T. Vicsek, A. Czirók, E. B. Jacob, I. Cohen, and O. Schochet, "Novel type of phase transitions in a system of self-driven particles," phys. Rev. Lett. vol. 75, no.6, pp. 1226-1229, Aug. 1995.

[15] R. Olfati-Saber and R. M. Murray, "Consensus problems in networks of agents with switching topology and time-delays," IEEE Transactions on Automatic Control, vol. 49, no. 9, pp. 1520-1533, 2004.

[16] A. Jadbabaie, J. Lin, and A. S. Morse, "Coordination of groups of mobile autonomous agents using nearest neighbor rules," IEEE Transactions on Automatic Control, vol. 48, no. 6, 2003.

[17] W. Ren, R. Beard, and E. Atkins, "A Survey of Consensus Problems in Multi-agent Coordination," Proceedings of 2005 American Control Conference, 2005.

[18] W. Ren and R. W. Beard, Distributed Consensus in Multi-vehicle Cooperative Control: Theory and Applications, Springer, 2007. 